$\underline{\text { Preprint typeset in JHEP style - HYPER VERSION }}$

hep-th/0702015

CPHT-RR005.0107

$\mathrm{ROM} 2 \mathrm{~F} / 07 / 04$

\title{
Non-perturbative and Flux superpotentials for Type I strings on the $\mathrm{Z}_{3}$ orbifold
}

\author{
Massimo Bianchi ${ }^{1}$ and Elias Kiritsis ${ }^{2,3}$ \\ ${ }^{1}$ Dipartimento di Fisica, Università di Roma "Tor Vergata" \\ I.N.F.N. - Sezione di Roma "Tor Vergata" \\ Via della Ricerca Scientifica, 1 - 00133 Roma, ITALY \\ ${ }^{2}$ CPHT, UMR du CNRS 7644, Ecole Polytechnique, CNRS, 91128 Palaiseau, FRANCE \\ ${ }^{3}$ Department of Physics, University of Crete, 71003 Heraklion, GREECE
}

\begin{abstract}
Non-perturbative effects are studied for Type I strings on the $\mathbf{Z}_{3}$ orbifold with Chan-Paton symmetry broken by Wilson lines. Generalizing previous analyses that have focussed on (bi)fundamentals, it is argued that (anti)symmetric representations of the resulting gauge group play a decisive role in generating an ADS-like superpotential in this and related cases. Non-perturbative corrections in the closed string moduli are only allowed if properly dressed with open string fields charged under the anomalous $U(1)$ of the orbifold. A general discussion of instanton effects in SYM and string theories is given. Non-perturbative superpotentials induced by both ED5's and ED1's are analyzed. The superpotential generated by closed string fluxes, viz. Scherk-Schwarz shifts (torsion), R-R 3 -form flux as well as non-geometric fluxes is derived. Some preliminary comments on the compatibility of the two kinds of superpotentials and the issue of moduli stabilization are presented.
\end{abstract}




\section{Contents}

1. Introduction and conclusions

2. General discussion of instanton effects

2.1 Instantons in supersymmetric gauge theories

2.2 Instantons in string theory

2.3 Compatibility of bulk isometries and non-perturbative effects 11

3. Review of type II $\mathrm{Z}_{3}$ orbifold 13

4. The $\mathrm{Z}_{3}$ orientifolds with Wilson lines 14

5. Non-perturbative effects in the $\mathrm{Z}_{3}$ orientifold 16

5.1 Wrapped Euclidean D5-branes 16

5.2 Wrapped D-strings 18

6. Fluxes 20

6.1 Scherk-Schwarz Torsion on $\mathbf{Z}_{3}$ orbifold 20

7. Concluding remarks 22

\section{Introduction and conclusions}

Vacuum configurations with open and unoriented strings [1] have received attention thanks to their remarkable phenomenological properties [2]. In such vacua the standard model gauge group is realized on stacks of intersecting or magnetized (co-isotropic) $D$-branes that give rise to massless chiral fermions at their intersections or as a result of the degeneracy of Landau levels [3]. Closed strings propagate in the bulk and mediate gravity and several other unobserved interactions through the exchange of scalar moduli (together with their superpartners).

The strong phenomenological appeal of orientifold vacua is mostly due to the fact that "bottom-up" approaches, 河, to realizing the Standard Model spectrum and interactions can be more or less implemented. In such approaches, one can assemble a local brane configuration with the required phenomenological properties, and postpone, global consistency conditions like tadpole cancellation. Indeed, in many cases this leads to configurations that can be eventually upgraded to bona fide vacua with a high degree of success ${ }^{1}$

\footnotetext{
${ }^{1}$ In the large scale computerized searches of [月, 6], local configurations that satisfy basic BCFT requirements have $\sim 1 \%$ probability to be completed into full vacua, by adjusting the hidden sector.
} 
Turning on internal fluxes both in the closed [7] and open string [8] sector one can achieve (supersymmetric) moduli stabilization in AdS space and then try to uplift the system to a metastable De Sitter vacuum with a tiny cosmological constant [9]. The last step is very delicate and requires taking carefully into account all perturbative and non-perturbative contributions to the potential simultaneously. Some of them are under control in full-fledged string theory (such as magnetic fluxes and NS-NS geometric and non-geometric fluxes), other (R-R fluxes) can be studied in the diluted flux approximation or in an effective supergravity description.

It is widely appreciated that a satisfactory implementation of supersymmetry breaking and moduli stabilization in string theory may not forgo a complete understanding of nonperturbative effects associated to (Euclidean) branes wrapping internal cycles [10, 11, 12].

An omnipresent, as well as crucial feature of orientifold vacua, is the existence of (potentially anomalous) $U(1)$ factors in the gauge group. Mixed anomalies of such factors have been shown to cancel both in 6D [13] and 4D vacua [14] by variants of the Green-Schwarz mechanism. However, it was subsequently appreciated, that there is a richer structure associated to anomalous $\mathrm{U}(1)$ 's that is not tied only to $4 \mathrm{D}$ anomalies [15, 16, 17, 18]. The presence of anomalous $\mathrm{U}(1)$, and their couplings require global knowledge of the orientifold ground-state, and in general cannot be successfully treated in local brane configurations ${ }^{2}$ Moreover, since the anomalous $U(1)$ symmetries are inextricably related to isometries of the manifold of closed string moduli, they are an integral part of any attempt to generate potentials and stabilize moduli. In initial stabilization setups, the role of anomalous U(1)'s was ignored. Their presence however is important in non-perturbative superpotentials, as the associated gauge invariance constraints the type of terms that appear, as it was first appreciated in [20] and recently studied in [12]. However, all related studies so far have been "local" and have avoided the global constraints of orientifolds.

The study of string instanton effects is still in its infancy. Several situations, especially with extended supersymmetry have been analyzed in the past (see 21] for a review) drawing mostly on non-perturbative dualities of string vacua with extended supersymmetry. However, the direct study of string instanton effects still remains an anecdotal subject (modulo some recent efforts [22], [12]).

In this paper we initiate their study for the simple case of the $\mathbf{Z}_{3}$ orbifold [24]. As we will see, (anti)symmetric representations of the Chan-Paton group will play a crucial role in these cases, generalizing previous analyses that have so far only considered (bi)fundamentals.

Depending on the stack of branes under consideration there are essentially two distinct classes of 'instantonic' branes. The first class involves branes that form bound states at threshold with the previous stack such as when they share $4 \mathrm{~N}-\mathrm{D}$ directions. The second includes branes that share $8 \mathrm{~N}-\mathrm{D}$ directions and accommodate only a chiral fermion at the intersection. The former are akin to gauge instantons [22]. The prototype is the $D 3, D(-1)$ system studied from various perspectives over the years [22]. The latter have only very

\footnotetext{
${ }^{2}$ Some exceptions to this statement exist, in special oriented ground-states where isolated stacks can appear, 19]. However, such examples remain to be seen whether they survive the cancellation of tadpole conditions.
} 
recently attracted some attention 12] and may eventually enjoy a field theory description in terms of octionionic instantons or hyper-instantons [23]. We will not pursue this point any further here but we will identify the role of this kind of stringy instantons in the vacuum we consider.

Including closed string fluxes we will have a complete picture of the full (super)potential and we will attempt a preliminary analysis of the resulting moduli stabilization problem.

In this paper we improve on previous analyses of instanton effects and non-perturbative superpotentials in several directions:

- We provide a clear classification of all $D$-brane instanton effects for Type I strings on the $\mathbf{Z}_{3}$ orbifold.

- We provide the correct form of various vertex operators corresponding to instanton modes, in particular the fermionic zero modes that are relevant.

- We include the effect of (anti) symmetric representations (eg. 6 of SU(4)) that are carefully avoided in previous works.

- We identify concrete rigid cycles (the combination of the exceptional divisors), In toroidal models where concrete efforts have been undertaken, all cycles are unfortunately sliding ones.

- We give a precise identification of the consistency conditions (Bianchi identities) $d G_{1}=F_{2}$ that constrain $E D$-string wrapping.

- We identify the (U(1)-charged) prefactors of instanton contributions to superpotential in two ways: by an instanton zero-mode counting and by $\mathrm{U}(1)$ neutrality (obviously the two are related)

- We finally give an expression for the superpotential with geometrical fluxes $\left(F_{3}\right.$ and torsion) as well as non-geometrical ones present.

The plan of the paper is as follows. We devote section to briefly review instanton effects in supersymmetric theories and discuss their stringy analogues. A crucial ingredient is played by anomalous $U(1)$ 's that become massive thanks to a generalization of the Green Schwarz mechanism in $D=4$. For this to happen a closed string axionic shift symmetry is gauged. This prevents the relevant axion from appearing in non-perturbative corrections if not properly dressed with open string fields charged under the anomalous $U(1)$. This is discussed in section 2.3. In section 3, we review basic facts about the geometry of the underlying orbifold and a schematic discussion of the quantum stringy corrections to the topological intersections. We then discuss in section th how to consistently include open and unoriented strings in the description. We also review the fate of the anomalous $U(1)$ ('s) and how discrete and continuous Wilson lines allow to conveniently break the Chan Paton gauge group. In section [, we specialize our instanton analysis to the case of the $\mathbf{Z}_{3}$ un-orientifold. We discuss non-perturbative superpotentials induced by both ED5's and ED1's. Then, in section 6 we discuss the superpotential generated by closed string fluxes, 
viz. Scherk-Schwarz shifts (torsion) and R-R 3-form flux included compatibly with the various projections. Finally, we conclude with some comments on T-duality and the issue of moduli stabilization.

\section{General discussion of instanton effects}

Instantons are classical solutions of the Euclidean field equations with finite action. Although, strictly speaking, they represent a set of zero measure in the space of field configurations, including quadratic fluctuations and integrating over the exact moduli may generate tiny, but new and important effects beyond the reach of perturbation theory. Unfortunately in pure Yang-Mills or QCD, a reliable computation of these effects is seriously hampered by IR divergences. The combination of instantons, holomorphy, anomaly and more recently duality considerations has proven to be an unprecedented tool in the investigation of supersymmetric theories. Instantons can generate non-perturbative corrections to the superpotential in $\mathcal{N}=1$ theories [25, 26, 27, 28], thus leading to the formation of chiral condensates that imply dynamical supersymmetry breaking in special cases by consideration of the Konishi anomaly. In $\mathcal{N}=2$ theories instantons correct the analytic prepotential encoded in the periods of an auxiliary Seiberg-Witten curve [32]. In $\mathcal{N}=4$ theories, thanks to the absence of $R$-symmetry anomalies, they interfere with perturbation theory and should account for non-perturbative corrections to anomalous dimensions of unprotected operators expected on the basis of $S$-duality [33].

The algebro-geometric construction of instantons in gauge theories, that goes under the name of ADHM construction after Atiyah, Drinfeld, Hitchin and Manin [34, finds an intuitive description in open string theory, whereby the gauge theory is realized on a certain stack of $D p$-branes and instantons are represented by a gas $D(p-4)$-branes within the previous stack [35, 22]. ADHM data are the lowest lying modes of the open strings connecting the $D(p-4)$-branes with one another or with the $D p$-branes. In a supersymmetric setting, these also account for fermionic (zero) modes. On top of their intrinsic beauty and elegance this kind of analysis has found a number of applications and proves crucial for our present purposes.

\subsection{Instantons in supersymmetric gauge theories}

Pure $\mathcal{N}=1$ supersymmetric Yang-Mills theories are expected to confine and to expose chiral symmetry breaking resulting from the formation of a chiral condensate for the gaugino. Although the exact spectrum of bound-states ('superglueballs') is only approximately known in the strong coupling regime at large $N$ thanks to generalizations of the AdS/CFT correspondence, the precise value of the chiral condensate in terms of the RG invariant scale $\Lambda$ can be indirectly derived by means of instanton calculus exploiting vacuum dominance. Indeed the chiral correlator

$$
\left\langle\lambda \lambda\left(x_{1}\right) \ldots \lambda \lambda\left(x_{N}\right)\right\rangle
$$

is dominated by instantons with instanton number $K=1$ that give rise to a constant result proportional to $\Lambda^{3 N}$ as expected on supersymmetry grounds and dimensional analysis. The 
dynamics of the massive "glueball" superfield $S=W^{\alpha} W_{\alpha}$ is governed by the VenezianoYankielowicz superpotential 26]

$$
W_{V Y}(S)=N S \log \frac{S}{c_{N} \Lambda^{3}}
$$

where $c_{N}$ is a constant depending on $N$ and on the scheme chosen (Strong Coupling vs Weak Coupling approaches). We will not address this subtle issue in the present paper.

If one includes matter in the form of chiral fields, their classical superfield equations get corrected by the Konishi anomaly [25]

$$
\frac{1}{4} \bar{D}^{2} \Phi_{I}^{\dagger} e^{g V} \Phi^{J}=\frac{\partial W}{\partial \Phi^{I}} \Phi^{J}+\delta_{I}^{J} \frac{g^{2}}{32 \pi^{2}} \operatorname{tr}_{\mathbf{R}} W^{2}
$$

This proves useful in setting the relative strength of the various allowed chiral condensates or, when this becomes impossible in a supersymmetric vacuum, in arguing for dynamical supersymmetry breaking. The existence of flat directions plays a crucial role in this respect. For instance consider $\mathcal{N}=1$ SQCD, whereby chiral multiplets in the $\mathbf{N}\left(Q_{i}\right)$ and $\mathbf{N}^{*}\left(\tilde{Q}_{i}\right)$ with $i=1, \ldots N_{f}$ are included. When $N=N_{f}+1$, instantons generate the Affleck, Dine, Seiberg superpotential [27]

$$
W_{A D S}=\frac{\Lambda^{2 N+1}}{\operatorname{det}(Q \tilde{Q})}
$$

In the absence of explicit mass terms this pushes the vacuum to infinity along a flat direction. If one turns on mass terms of the form

$$
W_{m}=\sum_{i j} m_{i j} Q^{i} \tilde{Q}^{j}
$$

the Konishi anomaly implies

$$
\sum_{i j} m_{i j}\left\langle Q^{i} \tilde{Q}^{j}\right\rangle=N_{f} \frac{g^{2}}{32 \pi^{2}}\langle\lambda \lambda\rangle
$$

Since the relevant chiral correlator in this case $\left(N=N_{f}+1\right)$ is

$$
\frac{g^{2}}{32 \pi^{2}}\left\langle\lambda \lambda\left(x_{0}\right) Q^{i_{1}} \tilde{Q}^{j_{1}}\left(x_{1}\right) \ldots Q^{i_{N_{f}}} \tilde{Q}^{j_{N_{f}}}\left(x_{N_{f}}\right)\right\rangle=\Lambda^{2 N+1} \epsilon^{i_{1} \cdots i_{N_{f}}} \epsilon^{j_{1} \cdots j_{N_{f}}}
$$

one finds

$$
\frac{g^{2}}{32 \pi^{2}}\langle\lambda \lambda\rangle=\Lambda_{L}^{3}=\left(N_{f} !\right)^{2} \frac{\Lambda^{2 N+1}}{\operatorname{det}(Q \tilde{Q})}=\left[\frac{\Lambda^{2 N+1}}{N_{f}^{N_{f}}} \operatorname{det} m\right]^{\frac{1}{N}}
$$

where $\Lambda_{L}$ is the RG invariant scale of the low-energy gauge theory along the flat direction. Moreover

$$
\left\langle Q^{i} \tilde{Q}^{j}\right\rangle=\left(m^{-1}\right)^{i j} \Lambda_{L}^{3}
$$

that clearly shows how the vacuum wanders to infinity when $\operatorname{det}(m)=0$. 
Using decoupling arguments one can generalize the analysis to the cases $N_{f} \leq N$, whereby the relevant one-instanton dominated correlator is

$$
\left(\frac{g^{2}}{32 \pi^{2}}\right)^{N-N_{f}}\left\langle\lambda \lambda\left(y_{1}\right) \ldots \lambda \lambda\left(y_{N-N_{f}}\right) Q^{i_{1}} \tilde{Q}^{j_{1}}\left(x_{1}\right) \ldots Q^{i_{N_{f}}} \tilde{Q}^{j_{N_{f}}}\left(x_{N_{f}}\right)\right\rangle=\Lambda^{3 N-N_{f}} \epsilon^{i_{1} \cdots i_{N_{f}}} \epsilon^{j_{1} \cdots j_{N_{f}}}
$$

where the exponent is not unexpectedly the one-loop $\beta$ function coefficient, $\beta_{1}=3 N-N_{f}$ in this case. In general

$$
\beta_{1}=3 \ell(\mathbf{A d j})-\sum_{I} \ell\left(\mathbf{R}_{I}\right)
$$

where $\ell(\mathbf{R})$ denotes the Dynkin index of the representation $\mathbf{R}$, normalized so that $\operatorname{tr}_{\mathbf{R}}\left(T^{a} T^{b}\right)=$ $\ell(\mathbf{R}) \delta^{a b}$.

At $N=N_{f}$ a baryonic branch opens up and for $[3 N / 2]>N_{f} \geq N+1$ SQCD admits an IR free dual 'magnetic' description. For $3 N>N_{f} \geq[3 N / 2]$ the theory enters the superconformal window. For $N_{f}>3 N$ the electric theory is trivial / free in the IR.

In more general (chiral) theories, one can use various symmetry arguments, including anomalous violation, in order to identify the relevant one-instanton dominated correlators. In the absence of flat directions, one can exploit vacuum dominance to extract the various chiral condensates compatibly with the Konishi anomaly. If this cannot be satisfied in a supersymmetric vacuum (in which the LHS vanishes!) one has to infer dynamical supersymmetry breaking. In the presence of flat directions the vacuum can wander to infinity in field space.

The rule of thumb for the one-instanton generation of a non-perturbative superpotential is the counting of fermionic zero-modes [25, 26, 27, 28]. This number should be two since

$$
L=\int d^{2} \theta W+\text { h.c. }
$$

In general there are $2 \ell_{\mathbf{A d j}}$ gaugino zero modes, e.g. $2 N$ for $S U(N)$, and $2 \sum_{I} \ell\left(\mathbf{R}_{I}\right)$ matter fermion zero-modes. If $\sum_{I} \ell\left(\mathbf{R}_{I}\right)<\ell_{\mathbf{A d j}}$, matter and gaugino zero-modes are lifted in pairs by Yukawa interactions

$$
L_{Y u k}=\sqrt{2} g \phi_{I}^{\dagger} \psi^{I} \lambda
$$

In particular for $\ell_{\mathbf{A d j}}-\sum_{I} \ell\left(\mathbf{R}_{I}\right)=1$, all matter fermion zero modes are lifted and only two gaugino zero-modes survive, the ones associated to the broken Poincaré susy. The non-perturbative superpotential acquires the strikingly simple form

$$
W_{n-p}=\Lambda_{L}^{3}=\frac{\Lambda^{3 \ell_{\mathbf{A d j}}-\sum_{I} \ell\left(\mathbf{R}_{I}\right)}}{\mathcal{H}(\Phi)}
$$

where $\mathcal{H}(\Phi)$ is a chiral gauge invariant, flavor singlet composite of mass dimension $\Delta_{\mathcal{H}}=$ $2 \sum_{I} \ell\left(\mathbf{R}_{I}\right)=2\left(\ell_{\mathbf{A d j}}-1\right)$ and $\Lambda_{L}$ is the RG invariant scale of the low-energy gauge theory along the flat direction.

In our stringy application we will need the above result for $G=S U(4) \approx S O(6)$ with 3 chiral multiplets in the 6 dimensional representation, that can be either viewed as the antisymmetric tensor of $S U(4)$ or as the vector of $S O(6)$. It easy to see that indeed 
$\ell_{\mathbf{A d j}}-\sum_{I} \ell\left(\mathbf{R}_{I}\right)=4-3=1$ in this case. Actually, as anticipated, an anomalous $U(1)$ will also play a crucial role in the string setting. Another, perhaps more interesting, case would be $G=S U(5)$ with two chiral multiplets in $\mathbf{5}+\mathbf{1 0}^{*}$. Once again $\ell_{\mathbf{A d j}}-\sum_{I} \ell\left(\mathbf{R}_{I}\right)=$ $5-2 \times(1 / 2+3 / 2)=1$ and an ADS-like superpotential of the form

$$
W_{A D S}=\frac{\Lambda^{11}}{\Phi_{\mathbf{5}}^{2} \Phi_{\mathbf{1 0}}^{6}}
$$

is generated by instantons. We will not delve into this case any further although it should admit a $D$-brane realization in string theory.

\subsection{Instantons in string theory}

World-sheet instantons in heterotic and type II theories have a long history [10]. They correspond to Euclidean fundamental string world-sheets wrapping non-trivial cycles of the compactification space and produce effects that scale as $e^{-R^{2} / \alpha^{\prime}}$. Depending on the number of supersymmetries and thus on the number of fermionic zero-modes, they may correct the two-derivative effective action or they can contribute to threshold corrections to higher derivative (BPS saturated) couplings. For type II compactifications on CY threefolds, preserving $\mathcal{N}=2$ supersymmetry in $D=4$, world-sheet instantons correct the special Kähler geometry of vector multiplets (type IIA) or the dual quaternionic geometry of hypermultiplets (type IIB). Mirror symmetry allows to relate the former to the tree level exact special Kähler geometry of vector multiplets in type IIB, that can be computed by algebraic methods in terms of the structure of the so-called chiral ring. For heterotic compactifications with standard embedding of the holonomy group $S U(3)$ in the gauge group, complex structure deformations $U^{a}$ (with $a=1, \ldots, h_{2,1}$ ) are governed by the same special Kähler geometry as in type IIB on the same CY threefold, that is not corrected by world-sheet instantons. Complexified Kähler deformations $T^{i}$ (with $i=1, \ldots, h_{1,1}$ ) are governed by the same special Kähler geometry as in type IIA on the same CY threefold, that is corrected by world-sheet instantons, or equivalently, as a result of mirror symmetry, by the same special Kähler geometry as in type IIB on the mirror CY threefold with $\tilde{h}_{2,1}=h_{1,1}$, that is tree level exact. For standard embedding, the Kähler metrics of charged supermultiplets in the $\mathbf{2 7}\left(C^{i}\right.$ with $\left.i=1, \ldots, h_{2,1}\right)$ and $\mathbf{2 7}^{*}\left(\tilde{C}^{a}\right.$ with $\left.a=1, \ldots, h_{1,1}\right)$ are simply determined by the ones of the neutral moduli of the same kind by a rescaling [29]. For non standard embeddings, the situation is not so obvious. In particular for some time it was believed that the resulting $\mathcal{N}=(2,0)$ SCFT would be destabilized by wordsheet instantons. More recently explicit examples have been constructed where world-sheet instanton effects conspire to cancel [30].

Before turning our attention to $D$-brane instantons, let us mention that Euclidean NS5-branes wrapping the 6-dimensional compactification manifold produce non-perturbative effects in $g_{s}$ (i.e. $e^{-c / g_{s}^{2}}$, reflecting the tension of $N S 5$-branes) that qualitatively correspond to 'standard' gauge and gravitational instantons [11].

$D$-brane instantons produce effects that scale as $e^{-c_{p} / g_{s}}$, reflecting the tension of $D p$ branes [1]. In type IIB on CY threefolds, ED(-1), ED1-, ED3- and ED5-brane instantons, obtained by wrapping holomorphic submanifolds of complex codimension $3,2,1$ 
and 0 respectively, correct the dual quaternionic geometry in combination with the above mentioned world-sheet (EF1-) and NS5-brane (EN5-) instantons. In type IIA ED2instantons ('membrane' instantons) wrapping special Lagrangian submanifolds, correct the dual quaternionic geometry, in combination with $N S 5$-brane (EN5-) instantons. Recall that the dilaton belongs to the universal hypermultiplet in both cases.

In type I, the presence of $\Omega 9$-planes severely restricts the possible homologically non trivial instanton configurations. Essentially only ED1- and ED5-branes are topologically stable. Other (Euclidean) branes can only be associated to instanton with torsion (Ktheory) charges. For other un-orientifolds the situation is similar and can be deduced by means of T-duality (e.g. for intersecting D6-branes one has two different kinds of ED2branes, for intersecting $D 3$ - and $D 7$ - branes one has $E D(-1)$ and $E D 3$-branes, etc).

As mentioned in the introduction there are essentially two extreme kinds of $E D$-brane instantons. When the $E D$-brane shares the equivalent of $4 \mathrm{ND}$ directions with a given stack of $D$-branes, it produces the stringy version of gauge instanton effects [22]. This situation is realized when the $E D$-branes wrap the same cycle as the background $D$-brane and is point-like in Euclidean space-time [12].

Although we only discuss $D 9$ with $E D 5$ and $D 9$ with $E D 1$, all other cases are essentially related by T-duality to this one we will focus on. To be precise, in a toroidal orbifold the most general case is a pair of magnetized $D 9$ and magnetized ED5 (the latter is point-like in the space-time directions). There are conditions that determine which 'magnetization' of the ED5 is compatible (due to supersymmetry) with the magnetization of the 'background' D9. An index theorem also fixes the number of zero modes and, as a result, determines whether the magnetized ED5 may produce a non zero F-term.

On the opposite side, when the ED-brane shares the equivalent of $8 \mathrm{ND}$ directions with a given stack of $D$-branes, it produces 'new' genuine stringy instanton effects that cannot be reproduced by standard gauge instantons [12]. In particular, in the Type I case, $E D 5$-branes qualitatively behave as gauge instantons for $D 9$-branes but as non-standard ('octonionic'?) instantons for $D 5$-branes (if present). On the other hand ED1-branes qualitatively behave as gauge instantons for $D 5$-branes wrapping the same internal cycles but as non-standard instantons for $D 9$-branes or for $D 5$-branes wrapping orthogonal cycles. We will consider precisely the effect of $E D 1$ on $D 9$ 's later on.

When world-volume (magnetic) fluxes are turned on a given stack of $D$-branes or a $E D$-branes the resulting effect is intermediate. We will not address this very interesting issue in the present paper since we will only work with isotropic (not coisotropic!) $D$ and ED-branes.

As shown in [22], $E D(p-4)$-branes within $D p$-branes precisely reproduce the instanton action, the ADHM data and as a result the instanton profile together with the associated zero-modes. One can thus proceed along the lines of the field theory analysis, i.e. identify the relevant one- or $K$ - instanton dominated amplitude and infer the form of the nonperturbative correction to the effective action.

The ADHM data correspond to strings connecting $E D(p-4)$-branes with one another or with $D p$-branes. We denote by $N$ the number of $D p$ branes and by $K$ the respective number of $E D(p-4)$. The corresponding bosonic vertex operators for $E D(p-4)-E D(p-4)$ 
strings $(K \times K$ of them $)$ are of the form

$$
V_{a}=a_{\mu} e^{-\varphi} \psi^{\mu} T_{K \times K}
$$

for the non dynamical gauge bosons, where $\mu$ denote the D-D space-time directions with no momentum $(p=0)$, and

$$
V_{\chi}=\chi_{i} e^{-\varphi} \psi^{i} T_{K \times K}
$$

for the non dynamical transverse scalars, where $i$ denote the internal directions longitudinal (NN) or orthogonal (DD) to the ED-branes not affected by twist / orbifold projections (if any).

The low-lying $E D(p-4)-D p$ strings ( $K \times N$ of them plus conjugate) admit bosonic vertex operators of the form

$$
V_{w}=\sqrt{\frac{g_{s}}{v_{p-3}}} w_{\alpha} e^{-\varphi} \prod_{\mu} \sigma_{\mu} S^{\alpha} T_{K \times N}
$$

where $\sigma$ are $Z_{2}$ twist fields and $S^{\alpha}$ is a spin field of a given chirality (left) along the $4 \mathrm{ND}$ directions and the overall normalization, suggested in [22] and then used in [12] is crucial in order to obtain the correct field theory limit.

The instanton action coincides with ( $K$ times) the gauge kinetic function since the $E D(p-4)$-branes under consideration wrap exactly the same (supersymmetric) cycle as the $D p$-branes. As such

$$
S_{\text {inst }, A}=f_{A}(S, T, U, Z, \Phi)
$$

can depend on moduli of various kinds: dilaton $(S)$, Kähler $(T)$, complex structure $(U)$, twisted $(Z)$, open string $(\Phi)$ both charged and neutral. For $D 9$ branes in orbifolds, for instance

$$
f_{D 9}=S+B_{I} Z^{I}+\Delta(T, U)+\ldots
$$

where $B_{I}$ denote the disk tadpole of $Z_{I}$ (twisted moduli) and $\Delta$ denote one-loop threshold corrections, which turn out to be a constant for the case of the $\mathbf{Z}_{3}$-orbifold we are interested in here.

By computing disk amplitudes with insertions of $V_{a}, V_{\chi}, V_{w}, V_{w^{\dagger}}$ and their superpartners, one can reconstruct the classical profiles needed to compute non-perturbative contribution to scattering amplitudes (see [22]).

The analysis of the other kind of instantons is different [12]. The prototype is the D9, D1 system whose (multi)-instanton configuration was first analyzed in [31]. The number of N-D directions is 8 in this case and the lowest lying modes of the open string stretched between the $N D 9$ 's and the $K D 1$ 's are massless fermions with a given chirality along the common two NN dimensions. In the standard case of type I strings there are 32 such chiral fermions $\left(\lambda^{A}\right)$ that precisely reproduce the gauge degrees of freedom of the 'dual' heterotic string. In addition from the 1-1 sector there are 8 transverse bosons $X^{I}$ in the $8_{v}$ of the $S O(8)$ R-symmetry group and as many Green-Schwarz type fermions $S^{a}$ of opposite chirality (say Left) in the $8_{s}$ giving rise to an $\mathcal{N}=(8,0)$ theory on the $D 1$ world-sheet. The 32 massless right-moving $\lambda^{A}$ are inert under the 8 left-moving susy $Q_{\dot{a}}$ in the $8_{c}$. 
After compactification to $D=4$ on a manifold with non-trivial holonomy some of the global supersymmetries are broken and the corresponding $D 1$ world-sheet theory changes accordingly. In particular the left-moving degrees of freedom include the surviving superspace variables $\Theta$ and $\bar{\Theta}$ descending from the GS fermions ${ }^{3}$

$$
V_{\Theta}=\Theta_{\alpha} S^{\alpha} \Sigma_{+3 / 2} e^{-\varphi / 2}
$$

where $S^{\alpha}$ is a dimension $1 / 4$ spin field in the space-time directions and $\Sigma$ is a dimension $3 / 8$ internal spin field. Four surviving (non-dynamical, no $p$ ) massless bosons,

$$
V_{a}=a_{\mu} e^{-\varphi} \psi^{\mu}
$$

corresponding to the motion along the four flat space-time directions, are always present. Extra massless bosons, corresponding to the motion along the internal directions, may appear depending on the possibility of deforming /sliding the cycle wrapped by the brane, however such cycles will not contribute to the superpotential. Rigid cycles would admit no such motions. The number of massless chiral fermions $\lambda$

$$
V_{\lambda}=\sqrt{g_{s}} \lambda_{R} e^{-\varphi / 2} S^{-} \prod_{\mu} \sigma_{(\mu)} \prod_{I} \sigma_{(I)}
$$

where $S^{-}$is a dimension $1 / 8$ right-handed spin field along the two NN directions, and $\sigma_{(\mu)}$ and $\sigma_{(I)}$ are $Z_{2}$ twist fields along the four space-time and as many internal ND directions, depends on the number of $D 9$ 's of a given type in the vacuum configuration that 'intersect' the D1's. Mutatis mutandis one can identify the relevant degrees of freedom for the other cases (e.g. ED3 in a background of D3, ED2 in a background of intersecting D6's, etc) 12 .

As described in [22], one has to integrate over the 'non-dynamical' modes living on the world-volumes of the $E D p$ under consideration. As a result one can generate nonperturbative corrections to the (super)potential. A comment however is in order. These effect are non-perturbative in that they scale as $e^{-T_{E D p} V_{E D p}}$. Since $T_{E D p} \approx 1 / g_{s}\left(\alpha^{\prime}\right)^{p+1 / 2}$ these effects are non-perturbative in $g_{s}$. Yet they a priori depend on different moduli (through the dependence of $V_{E D p}$ on various $Z$ 's) from the ones that appear in the gauge kinetic function(s) so they cannot in general be identified with 'standard instantons'. In fact one can envisage the possibility of turning on magnetic fluxes on the world-volume of the $E D p$ that allow one to interpolate ${ }^{4}$ between one kind of $E D p$ (e.g. a 'standard instanton') [22 and a different kind of $E D p$ (e.g. a new stringy instanton) [12].

As shown in [12], elaborating on the $g_{s}$ power counting introduced in [22], the relevant diagrams are disks with insertions of the non-dynamical vertex operators $V_{\Theta}$ (connecting $E D p$ 's with themselves) and $V_{\lambda}$ (connecting $E D p$ 's with the background $D p^{\prime}$ ) with or without insertions of the dynamical vertex operators $V_{A}$ etc corresponding to the massless excitations of the vacuum configuration of (intersecting / magnetized ) unoriented $D$-branes.

\footnotetext{
${ }^{3}$ For an instanton contribution to the superpotential the $\bar{\Theta}$ should be either massive or projected out. We will confirm this in section 5.2.

${ }^{4}$ This interpolation is discontinuous due to the quantization of the magnetic fluxes but may become quasi-continuous at large volumes.
} 
Disks without insertions of the latter type yield after exponentiation the 'instanton action' (including interactions of the pseudo zero-modes $\lambda$ 's etc). Disks with one dynamical vertex produce the classical profile that is needed for the computation of the non-perturbative amplitudes. Disks with more insertions contribute to higher-order corrections that can be neglected at first and consistently incorporated later on by symmetry arguments. One loop diagrams with no insertions should produce subtle numerical prefactors that can conspire so as to cancel a given type of non-perturbative F-terms [30].

For a supersymmetric instanton there are two $\Theta$ zero-modes. Suppose that one also has $2 n \lambda$ zero-modes. Then one can compute an F-term either by a combination of $n$ disks, each with two $\lambda$ insertions, out of which either $n-2$ of them accommodate one $V_{\phi}$ and two of them one $V_{\psi}$ each, or $n-1$ of them accommodate one $V_{\phi}$ and two of them one $V_{F}$ (vertex operators for auxiliary fields can be written in a non BRS invariant form that give anyway sensible results). Integrating over $\Theta$ 's and $\lambda$ 's yield a superpotential term of the form

$$
W=e^{-T_{E D p} V_{E D p}(Z)} \Phi^{n}
$$

where the notation is schematic in that $\Phi^{n}$ denotes a gauge invariant monomial of degree $n$ in the superfields $\Phi^{i}=\phi^{i}+\Theta \psi^{i}+\Theta^{2} F^{i}$ and $Z$ denote moduli fields whose dependence is tightly constrained by geometric and other symmetry considerations.

\subsection{Compatibility of bulk isometries and non-perturbative effects}

On general grounds a chiral field $Z$ whose pseudoscalar axionic components $\zeta=\operatorname{Im} Z$ shifts under some local anomalous $U(1)$ cannot appear as such in a (super)potential term. However it can appear dressed with other chiral fields that are charged under the $U(1)$. $U(1)$ invariance puts stringent constraints on the form of the possible superpotential terms. Since the axionic shift is gauged it must be a symmetry of the kinetic term. This is only possible when no non-perturbative (world-sheet or $D$-brane instanton) correction spoils the tree level (in fact perturbative) PQ symmetry of the Kähler potential. In turn this means that the gauging procedure corresponds to turning on fluxes such that the potential instanton corrections in $Z$ are in fact disallowed. In practice, this means the corresponding wrapped brane is either anomalous (à la Freed Witten) [36] or destabilized due to the flux [37]. In this respect chiral fields that appear in superpotential terms tend to have 'quasi-canonical' kinetic terms compatible with their continuous shift symmetries that are gauged.

This state of affairs has been checked in various cases [38] (e.g. ED3 in flux compactifications with $D 3$ and $D 7$ 's) and will also hold in the case we are going consider i.e. the $\mathbf{Z}_{3}$ orbifold with Wilson line breaking $U(12) \times S O(8)$ to $U(4) \times G_{H}$ with $G_{H}$ a hidden gauge group such as $U(4)_{C F T}^{3}$ or $U(4)_{\mathcal{N}=4}$ or $U(1)^{4}$.

In general, the Bianchi identities for the 'total' R-R field strength $G=\sum_{p} G_{p}$ is encoded in

$$
D G=\Pi[\text { branes }] \wedge e^{F}
$$

where $\Pi[$ branes $]=\sum_{p} \Pi_{9-p}[D p]$ denotes a formal sum of $(9-p)$-forms along the directions orthogonal to the branes present in the background, $D=d+\mathcal{T}+H$ where $\mathcal{T}$ is the geometric 
torsion à la Scherk-Schwarz and $H$ is the NS-NS 3-form field strength. ${ }^{5}$ For the lowest type IIB R-R forms one gets

$$
d G_{1}+\mathcal{T} \circ G_{1}=F_{2} \wedge \Pi_{0}(D 9)+\Pi_{2}(D 7)
$$

and

$$
d G_{3}+\mathcal{T} \circ G_{3}+H_{3} \wedge G_{1}=F_{2} \wedge F_{2} \wedge \Pi_{0}(D 9)+F_{2} \wedge \Pi_{2}(D 7)+\Pi_{4}(D 5)
$$

and so on, were we denoted the action of the geometric torsion on forms as

$$
\mathcal{T} \circ A_{p} \equiv(p+1) \mathcal{T}^{s}{ }_{\left[\mu_{1} \mu_{2}\right.} A_{\left.\mu_{3} \cdots s \cdots m_{p+1}\right]}
$$

Barring torsion $(\mathcal{T}=H=0)$ and D7-branes $(2.26)$ yields

$$
d G_{1}=F_{2}
$$

Integrating this on the closed world-volume of a D-string we obtain

$$
\int F_{2}=\int d G_{1}=0
$$

In general the axionic shifts are given by

$$
\delta \beta_{R-R}^{I}(x)=\alpha^{a}(x) \int_{\mathcal{C}_{I}} \operatorname{tr}\left(F_{a}\right)
$$

where $\mathcal{C}_{I}$ represents a basis of 2-cycles dual to the harmonic 2-forms $\omega_{I}(y)$ that appear in the expansion of the R-R 2-form

$$
C_{R-R}(x, y)=\beta_{R-R}^{I}(x) \omega_{I}(y)+\ldots(\text { massive })
$$

This means one cannot wrap an $E D$-string on any cycle $\mathcal{C}$ such that

$$
\int_{\mathcal{C}} \operatorname{tr}\left(F_{a}\right) \neq 0
$$

i.e. around the cycle dual to the R-R axion whose shift symmetry is gauged. This remains true even if $G_{1}$ and $F_{2}$ are odd under $\Omega$ (worldsheet parity, i.e. in unoriented theories with $D 9$ and $D 5$ ) very much as in the 'standard' construction with $D 3$ and $D 7$ branes the presence of $H_{3}$ and $F_{3}$ fluxes (" $G_{3}$ " fluxes) obstructs some $E D 3$-brane instantons even if the fluxes are odd under $\Omega^{\prime}=\Omega(-)^{F_{L}} \sigma$. For the $\mathbf{Z}_{3}$ orbifold we will momentarily see that $\mathcal{C}$ is a democratic linear combination of the 27 twisted cycles corresponding to collapsed $P^{2}$ s at the orbifold points.

\footnotetext{
${ }^{5}$ We neglect from this discussion the presence of the curvature terms in the WZ action as well as the non-geometric fluxes $\mathcal{Q}$ and $\mathcal{R}$ for simplicity. The relevant non-geometric fluxes will be restored later on.
} 


\section{Review of type II $\mathrm{Z}_{3}$ orbifold}

To construct the $\mathbf{Z}_{3}$ orbifold we act on the three complex coordinates of the $\mathbf{T}^{6}$ torus, $z^{I}$, $I=1,2,3$ as

$$
z_{I} \rightarrow \omega z_{I} \quad, \quad \omega=e^{2 \pi i / 3}
$$

To be a symmetry of $\mathbf{T}^{6}$, we must constraint the metric and the NS-NS two-form to be

$$
d s^{2}=G_{I \bar{I}} d z^{I} d \bar{z}^{\bar{I}} \quad, \quad B_{2}=B_{I \bar{I}} d z^{I} \wedge d \bar{z}^{\bar{I}}
$$

The $3 \times 3$ complex matrix $G_{I \bar{I}}$ is hermitian while $B_{I \bar{I}}$ is anti-hermitian. The $\mathbf{Z}_{3}$ action is chosen so that the holomorphic three-form $d z^{1} \wedge d z^{2} \wedge d z^{3}$ is invariant. Therefore the $\mathbf{Z}_{3}$ orbifold is a (singular) CY three-fold.

Compatibility with the $\mathbf{Z}_{3}$ projection freezes out all the complex structure deformations but allows 9 untwisted deformations of the Kähler structure $d z^{I} \wedge d \bar{z}^{\bar{J}}$, so that $h_{1,1}^{\text {untw }}=9$, while $h_{2,1}^{\text {untw }}=0$. The complex untwisted Kähler moduli are $G_{I \bar{J}}+B_{I \bar{J}}$. They can both be expanded in the standard basis of hermitian matrices $\mathcal{H}_{i j}$ as

$$
G=t_{i j} \mathcal{H}^{i j} \quad, \quad B=i b_{i j} \mathcal{H}^{i j}
$$

The resulting moduli space of untwisted complexified Kähler moduli is

$$
\mathcal{M}_{(1,1)}^{u n t w}=\frac{S U(3,3)}{S U(3) \times S U(3) \times U(1)}
$$

It is a special Kähler manifold with (holomorphic) prepotential

$$
\mathcal{F}_{\text {unt }}=\operatorname{det}[T]=\frac{1}{3 !} \epsilon^{I_{1} I_{2} I_{3}} \epsilon^{J_{1} J_{2} J_{3}} X_{I_{1} J_{1}} X_{I_{2} J_{2}} X_{I_{3} J_{3}} \quad, \quad X_{I J}=t_{i j}+i b_{i j}
$$

The associated Kähler potential is given by the special geometry formula

$$
\begin{gathered}
K_{\text {unt }}=-\log \left[2\left(\mathcal{F}_{\text {unt }}+\overline{\mathcal{F}}_{\text {unt }}\right)-\sum_{I J}\left(X_{I J}-\bar{X}_{I J}\right)\left(\frac{\partial \mathcal{F}_{\text {unt }}}{\partial X_{I J}}+\frac{\partial \overline{\mathcal{F}}_{\text {unt }}}{\partial \bar{X}_{I J}}\right)\right] \\
=-\log [\operatorname{det}[\operatorname{Re}[X]]]
\end{gathered}
$$

where the $X_{I J}$ are the inhomogeneous Kähler coordinates. The two-forms dual to the moduli $X_{I J}$ are $\omega_{I J}=d z^{I} \wedge d \bar{z}^{J}$ in one-to-one correspondence with the non-trivial twocycles of the torus, with intersection form

$$
\int \omega_{I_{1} J_{1}} \wedge \omega_{I_{2} J_{2}} \wedge \omega_{I_{3} J_{3}}=\epsilon_{I_{1} I_{2} I_{3}} \epsilon_{J_{1} J_{2} J_{3}}
$$

The associated four-forms are $\omega^{I J}=\epsilon^{I I_{1} I_{2}} \epsilon^{J J_{1} J_{2}} d z^{I_{1}} \wedge d z^{I_{2}} \wedge d \bar{z}^{J_{1}} \wedge d \bar{z}^{J_{2}}$.

$\mathbf{Z}_{3}$ has 27 fixed points corresponding to as many 'exceptional divisors', $E_{i}, i=1,2, \cdots, 27$. They are codimension-one complex submanifolds which are homologically non trivial. There are as many twisted $(1,1)$-forms, so that $h_{1,1}^{\text {twist }}=27$, while $h_{2,1}^{\text {twist }}=0$. There are three fixed points per two-plane so that we will label the 27 fixed points by $f_{i}$. 
The orbifold is resolved by excising a small neighborhood around the fixed points and gluing in $\mathbf{Z}_{3}$ Eguchi-Hanson-like balls each with Euler number $\chi=3$. Since the original torus has $\chi=0$ and each excised point has $\chi=1$ we obtain the total Euler number

$$
\chi\left(\mathbf{T}^{6} / \mathbf{Z}_{3}\right)=\frac{0-27}{3}+27 \cdot 3=72
$$

The two-forms dual to the exceptional cycles $\omega^{i}$ have the non-trivial intersection

$$
\int \omega^{i} \wedge \omega^{i} \wedge \omega^{i}=1 \quad, \quad \forall i
$$

while all other intersections between different fixed points, or with the untwisted ones, vanish. We will denote the 27 associated complex twisted moduli by $Z_{i}$.

The parent type IIB theory enjoys local $\mathcal{N}=2$ supersymmetry. In addition to the supergravity multiplet and the universal hypermultiplet, whose four scalars can be identified with the dilaton, the R-R axion, the NS-NS 2-form and the R-R 2-form both dual to axions, the massless spectrum contains $36=h_{1,1}^{\text {untw }}+h_{1,1}^{\text {twist }}$ hypermultiplets. Their scalar components are the Kähler deformations of the metric which are tri-complexified by the NS-NS 2-form and the R-R 2-form and the self-dual 4-form.

The unoriented projection preserves local $\mathcal{N}=1$ supersymmetry, thus eliminating the R-R graviphoton and one (linear combination of the) gravitini. Each (linear/) hypermultiplet produces a (linear/) chiral multiplet. The standard $\Omega$ projection retains the dilaton, the metric and the R-R 2-form, but one can envisage different (not necessarily) equivalent truncations. For instance, after 6 T-dualities one ends up with an essentially equivalent theory with $\Omega 3$-planes, that retains the dilaton, the $\mathrm{R}-\mathrm{R}$ axion, the metric and the $\mathrm{R}-\mathrm{R}$ 4-form.

A relative of the $\mathbf{Z}_{3}$ orbifold is obtained by acting with another $\mathbf{Z}_{3}$ transformation that rotates the coordinates $z^{1}, z^{2}$ as $z^{i} \rightarrow \omega z^{i}$. There are two options here, the free action or the non-free action. In both cases out of the 9 untwisted moduli $X_{I J}$ only three survive the new projection: $T_{11}, T_{22}, T_{33}$. In the twisted sector the situation depends on the action. Most interesting for us will be the free action where the the extra $\mathbf{Z}_{3}$ transformation is accompanied by a $\mathbf{Z}_{3}$ translation on the third torus along the lattice. Because the old fixed points remain fixed, the new orbifold has the same twisted sector as before, and the same number of massless twisted moduli. On the other hand the non-free $\mathbf{Z}_{3} \times \mathbf{Z}_{3}^{\prime}$ orbifold has 81 fixed points.

In the free case, the associated prepotentials as well as Kähler potentials can be obtained directly by restriction from those of the $\mathbf{Z}_{3}$ orbifold studied above. In this last case, the string instanton corrections to the prepotential have been studied in 40.

\section{The $\mathrm{Z}_{3}$ orientifolds with Wilson lines}

The standard $\Omega$-projection of the closed string spectrum generates R-R tadpoles that can be canceled by introducing $D 9$-branes and their (unoriented) open string excitations. Denoting by $\gamma_{\mathbf{z}_{3}}$ the projective embedding of the orbifold group in the Chan-Paton group, 
twisted tadpole cancellation requires $\operatorname{Tr}\left(\gamma_{\mathbf{Z}_{3}}\right)=-4$ in addition to the 'standard' untwisted tadpole condition $\operatorname{Tr}(\mathbf{1})=32$ [24]. Imposing $\gamma_{\mathbf{Z}_{3}}^{3}=1$ and $\gamma_{\mathbf{Z}_{3}}^{\dagger}=\gamma_{\mathbf{Z}_{3}}^{-1}$, allows to set $\gamma_{\mathbf{Z}_{3}}=\left(\mathbf{1}_{N \times N}, \omega \mathbf{1}_{M \times M}, \bar{\omega} \mathbf{1}_{\bar{M} \times \bar{M}}\right)$ so that

$$
N+M+\bar{M}=32 \quad N+\omega M+\bar{\omega} \bar{M}=-4 \quad M=\bar{M}
$$

yielding $N=8, M=\bar{M}=12$. Due to the $\Omega$ projection the resulting gauge group is $S O(8) \times U(12)$. In addition one has three generations of $(\mathbf{8}, \mathbf{1 2})_{+1}$ plus $\left(\mathbf{1}, \mathbf{6 6}^{*}\right)_{-2}$, resulting from the breaking of $\mathcal{N}=4 \mathrm{SYM}$ to $\mathcal{N}=1 \mathrm{SYM}$ plus three chiral multiplets, all transforming the same way under $\mathbf{Z}_{3}$. The $U(1)$ is anomalous, i.e. $t_{3} \neq 0$ where the mixed anomaly trace is

$$
t_{3} \equiv \operatorname{Tr}\left[Q_{f} T^{a} T^{a}\right]=\sum_{f} Q_{f} \ell\left(\mathbf{R}_{f}\right)
$$

with $f$ running over chiral (L) fermions with charge $Q_{f}$ and $\ell\left(\mathbf{R}_{f}\right)$ is the Dynkin index of the representation $\mathbf{R}_{f}$ of the non-abelian group $(G=S O(8) \times U(12))$ the fermions belong to. The generalized GS mechanism entails a mixing between $V$, the $\mathrm{U}(1)$ vector superfield, and a 'democratic' combination of all twisted chiral multiplets. Indeed, if we define $Z=\sum_{i=1}^{27} Z_{i}$ then

$$
Z+\bar{Z} \rightarrow Z+\bar{Z}-M V
$$

where $M \approx M_{s}$ is a mass parameter (computed in [16]), so that under

$$
V \rightarrow V+i \alpha-i \bar{\alpha}
$$

one has

$$
Z \rightarrow Z+i M \alpha
$$

Indicating the gauge kinetic functions of the non abelian gauge groups by

$$
f_{a}(T, S ; Z ; C, A)=f_{a}(T, S)+C_{a} Z+\ldots
$$

anomaly cancellation requires

$$
M_{a} C_{a}=t_{3, a}
$$

[14].

Chiral multiplets $C_{i}^{I r}$ in the $(\mathbf{8}, \mathbf{1 2})_{+1}$ and $A_{[r s]}^{I}$ in the $\left(\mathbf{1}, \mathbf{6 6}^{*}\right)_{-2}$ interact via the tree level superpotential [24]

$$
W(C, A ; T, S ; Z)=\frac{1}{3 ! 2 !} Y(T, S ; Z) \epsilon_{I J K} \delta^{i j} C_{i}^{I r} C_{j}^{J s} A_{[r s]}^{K} \quad .
$$

In the T-dual descriptions in terms of D3-branes, when all the branes are at the same fixed point, $Y(T, S, Z)$ should only depend on the overall volume multiplied by $e^{-\phi}$. However if regular $D 3$-branes move into the bulk, there could be open string instanton contributions too between far away branes, as can be checked by explicit computation of a disk diagram. Dependence on the closed string (un)twisted moduli is highly constrained by the axionic (shift) symmetries of the axions contained in $T, S$ and $Z$ (we have collectively labeled by $T$ the untwisted moduli and $Z$ the twisted ones). 
Consistently with the above picture, non-perturbative $F / D$-string instanton corrections are allowed in this case but no perturbative correction that would spoil the universal axion $(\operatorname{Im} S)$ PQ symmetry.

Clearly the physical Yukawas are renormalized as a consequence of the renormalization of the Kähler potential. Higher order terms in the neutral combination $C C A$ can appear however. Yet terms containing $\operatorname{Pfaff}(A)$ have a nontrivial $U(1)$ (anomalous) charge and can only be produced non-perturbatively, if at all, because there is no way to contract the indices in a cyclic fashion to produce $\epsilon^{r_{1} \ldots r_{12}}$ 41, 42, 6]. $U(1)$ symmetry prevents them from appearing in perturbation theory even from non-planar graphs. The situation may change if one takes into account the non-trivial $\mathrm{U}(1)$ gauge transformation properties of $Z$.

In order to study this possibility, it is convenient to turn on ('continuous') Wilson lines $\gamma_{W}$ along the flat directions of the potential and generically break $S O(8) \times U(12)$ to $U(4)_{f p} \times U(1)^{4}$ [4, 45]. Special ('discrete') choices of the Wilson lines correspond to symmetry enhancement [1]. For instance $\gamma_{W}$ commuting with $\gamma_{\mathbf{Z}_{3}}$ breaks $S O(8) \times U(12)$ to $S O(8-2 n) \times U(12-2 n) \times U(n)^{3}$. In particular for $n=4$ one gets $U(4)_{f p} \times U(4)^{3}$ coupled to 3 generations of chiral matter in the $\left(\mathbf{6}_{-2} ; \mathbf{1}_{0}, \mathbf{1}_{0}, \mathbf{1}_{0}\right)$ plus $\left(\mathbf{1}_{0} ; \mathbf{4}_{+1}, \mathbf{4}_{-1}^{*}, \mathbf{1}_{0}\right)$, $\left(\mathbf{1}_{0} ; \mathbf{1}_{0}, \mathbf{4}_{+1}, \mathbf{4}_{-1}^{*}\right),\left(\mathbf{1}_{0} ; \mathbf{4}_{-1}^{*}, \mathbf{1}_{0}, \mathbf{4}_{+1}\right)$. Notice that the $U(4)^{3}$ part correspond to a decoupled conformal theory living on a stack of 4 regular branes i.e. 4 branes together with their 6 images under $\mathbf{Z}_{3}$ and $\Omega$. In the T-dual description in terms of $D 3$-branes, the latter are located at two fixed points different from the origin which are mapped into one another by $\Omega$. One can further break $U(4)^{3}$ to $U(4)_{\text {diag }}$ with 3 adjoint chiral multiplet thus reconstructing the field content of $\mathcal{N}=4$ SYM. Finally by turning on VEV's for the six adjoint scalars one generically breaks the group to $U(1)^{4}$. One can turn on internal magnetic fields along these three $U(1)$ 's. We refrain from doing so here.

The case of the $\mathbf{Z}_{3} \times \mathbf{Z}_{3}^{\prime}$ orbifold where the second $\mathbf{Z}_{3}$ action is free is similar. Indeed as long as the size of the Scherk-Schwarz one-cycle is non-zero, the extra $\mathbf{Z}_{3}$ acts as a simple projection in the low energy sector. Moreover, it does not induce additional tadpoles and therefore the open sector is similar to the $\mathbf{Z}_{3}$ one, module the overall extra $\mathbf{Z}_{3}$ projection 46].

\section{Non-perturbative effects in the $\mathrm{Z}_{3}$ orientifold}

We are ready to discuss non-perturbative effects in the $\mathbf{Z}_{3}$ orientifold. We will start with the effect induced by wrapped Euclidean D5-branes (ED5-branes) that are expected to reproduce gauge instanton effects. We then consider the effects due to wrapped Euclidean D1-branes ED-strings.

\subsection{Wrapped Euclidean D5-branes}

At the point where $G=U(4)_{f p} \times U(4)_{d i a g}$, with $3 \mathbf{6}_{-2}$ for the first factor, instanton calculus is reliable. This is due to the fact that along the flat directions the gauge group is broken in such a way that no light charged matter survives. Indeed along the flat directions where the group is broken to $G_{L}=S O(3)$, instantons in the resulting pure $\mathcal{N}=1$ theory induce gaugino condensation with $W=\Lambda_{L}^{3}$. The matching condition between $\Lambda_{L}$ and $\Lambda$, the RG 
invariant scales of the low and high energy theories respectively, allows one to identify this superpotential with

$$
W=\Lambda_{L}^{3}=\frac{\Lambda^{9}}{\operatorname{det}_{I, J}\left(\delta^{a b} A_{a}^{I} A_{b}^{J}\right)}
$$

where $A_{a}^{I}=\frac{1}{2} \Gamma_{a}^{r s} A_{r s}^{I}$ with $a=1, \ldots, 6$ are the three chiral multiplets in the 6 of $S U(4)$, and $\Gamma_{a}^{r s}$ are Weyl blocks of 6 -d Dirac matrices. In general the argument applies whenever $\ell_{A}-\sum_{C} \ell_{C}=1$, where $A$ denotes the adjoint representation and $C$ runs over chiral multiplets. Indeed, in our case $\ell_{A}=4$ and $\sum_{C} \ell_{C}=3$.

In string theory one expects (up to an overall numerical factor)

$$
W(S, T, Z ; A)=\frac{e^{f(S, T, Z)}}{\mathcal{H}(A)}
$$

where

$$
f(S, T, Z)=f_{\text {tree }}(S, Z)+f_{1-\text { loop }}(T, Z)
$$

is the gauge kinetic function with ${ }^{6}$

$$
f_{\text {tree }}(S, Z)=S+C Z
$$

while

$$
f_{1-\text { loop }}(T, Z=0)=f_{1}
$$

a constant independent of $T$ 's as originally observed in [47] and confirmed in [48] following previous work on heterotic orbifolds [29]. The $Z$ dependence is harder to determine. $S$ independence is from loop counting. One can indeed check that under simultaneous $U(1)$ transformations of the $A$ 's and shift of $Z$, the superpotential $W$ is invariant. Indeed based on the mixed $U(1) \times S U(4)^{2}$ anomaly

$$
t_{144}=-2 \times 3 \times 2=-12
$$

one deduces that $Z$ must shift as

$$
Z \rightarrow Z-\frac{12}{C} i \alpha
$$

as shown in (4.6,4.7). This is exactly what is needed to cancel the transformation of the denominator generated by

$$
A \rightarrow e^{-2 i \alpha} A
$$

As previously stated in general terms, the instanton action is given by the worldvolume of an ED5 wrapping the entire orbifold and this is exactly given by the gauge kinetic function of the $D 9$ branes, including the shift $C Z$. Notice that in the present case, generation of a non-vanishing AdS-like superpotential heavily relies on the presence of the doubly-charged anti-symmetric representations $\left(\mathbf{6}_{-2}\right)$ of the $U(4)$ Chan-Paton group.

\footnotetext{
${ }^{6} \mathrm{~A}$ dependence of the holomorphic gauge kinetic function on the open string moduli $A$ would entail, for reasons of $\mathrm{U}(1)$ gauge invariance, a further exponential dependence on $Z$. This would imply "instanton corrections for instanton corrections". We deem that such dependence is unlikely.
} 
In a recent paper [49], a detailed stringy derivation of the ADS superpotential has been given for the case of SQCD with gauge group $S U\left(N_{c}\right)$ with $N_{f}=N_{c}-1$ massless flavours or $S p\left(2 N_{c}\right)$ with $2 N_{f}=2 N_{c}$ flavours. The case $S O\left(2 N_{c}\right)$ with $N_{f}=N_{c}$, we focussed on above for $N_{c}=6$, was only touched upon. In the construction of 49] the gauge theory is realized on a stack of D6-branes and the flavour symmetry is generated by another stack of D6-branes intersecting the previous one in a non chiral fashion. The relevant instanton is an ED2 wrapping the same cycle as the stack of $N_{c}$ D6-branes. By careful integrating the supermoduli the precise form of the ADS superpotential was reproduced in the field theory limit. In our case the gauge theory is realized with branes at a singularity and we have determined the form of the ADS-like superpotential by holomorphy, dimensional analysis, $U(1)$ anomaly (fermion zero-mode counting) and flavour symmetry. We leave it as an open problem to derive the ADS-like superpotential directly from a full-fledged string computation along the lines of [49]. In order to do so one has to properly integrate the supermoduli that comprise massless strings stretching from ED5 to the D9's and those of the ED5 itself that should support a $U(1)$ Chan-Paton group that should enhance to $S p(2)$.

\subsection{Wrapped D-strings}

We would now like to discuss non-perturbative effects induced by wrapping ED1 around topologically non-trivial two-cycles. This configuration was first considered in [31, where the ED1 instanton corrections to the $F^{4}$ and $R^{4}$ couplings were computed in the toroidally compactified type-I theory. These corrections were originally obtained by heterotic/type I duality from the one-loop string instanton corrections in the heterotic string but subsequently justified from the ED1 instanton point of view. This computation also gave a detailed account of the multi-instanton contributions and their subtleties.

In our case we will first show that unlike the $\Theta$ zero-modes present and described in section 2.2, the $\bar{\Theta}$ zero modes are absent. We start with the supersymmetry in $\mathrm{D}=10$ for $D_{9}$-branes: the 16 of $\mathrm{SO}(10)$. Adding ED1's in flat space-time we decompose $16 \rightarrow$ $8_{s}^{+1 / 2}+8_{c}^{-1 / 2}$ under $S O(8) \times S O(2)$. One of the two spinors is projected out so we assume that $8_{c}^{-1 / 2}$ remains. It generates $\mathcal{N}=(8,0)$ world-sheet supersymmetry with respect to which which the fermions $\lambda$, in the D9-ED1 sector are inert. After compactification on the orbifold, $\mathrm{SO}(8)$ is broken at least to $S O(4)_{\text {Min }} \times S O(4)_{\text {int }}$ where $S O(4)_{\text {Min }}$ is the Lorentz symmetry of flat space-time Therefore, $8_{c} \rightarrow\left(2_{L}, 2_{L}\right)+\left(2_{R}, 2_{R}\right)$. In order to have a surviving supersymmetry the orbifold projection $g=\exp \left(i w_{i} J_{i}\right)$ must be such that $w_{1}+w_{2}+w_{3}=0$. The surviving spinors are the lowest components of $\left(2_{L}\right)_{\text {int }}$ i.e. $(-1 / 2,-1 / 2)$ which when combined with the $-1 / 2$ helicity with respect to the the 'worldsheet' $\mathrm{SO}(2)$ yield $-1 / 2 w_{1}-1 / 2 w_{2}-1 / 2 w_{3}=0$. Clearly choosing a different projection with $\pm w_{1} \pm w_{2} \pm w_{3}=0$ a different but unique internal spinor component will survive. As a result only one $S O(4)_{M i n}$ chirality of the supersymmetry survives i.e. $\left(2_{L} ;(-1 / 2,-1 / 2) ;-1 / 2\right)$ of $S O(4) \times S O(4) \times S O(2)$. Obviously the second $\mathrm{SO}(4)$ is not a symmetry, but it is helpful in the above decomposition. In a smooth CY the four supersymmetry charges are $Q_{\alpha}=S_{\alpha} \eta, \bar{Q}_{\dot{\alpha}}=C_{\dot{\alpha}} \eta^{\dagger}$ where $\eta$ and $\eta^{\dagger}$ are the two covariantly constant spinors of opposite $\mathrm{SO}(6)$ chirality (or U(1) charge, under $S O(6) \rightarrow S U(3) \times U(1)$ ). Only one of the two has the correct chirality under the $\mathrm{SO}(2)$ of the ED1 world-sheet. 
In order to determine which kind of superpotential term is generated one has to count the number of fermionic zero modes $\lambda$ 's stretching from the $E D 1$ to the background $D 9$ 's. Depending of the 2 -cycle $\mathcal{C}$ wrapped by the $E D 1$, i.e. on the restriction $\left.V\right|_{\mathcal{C}}$ of the vacuum gauge bundle $V$ to $\mathcal{C}, \lambda$ 's transforming in the $\boldsymbol{4}_{+1}$ of $U(4)_{D 9}$ may appear. These can couple at the disk level with the scalar component $a^{I}$ of the multiplet $A^{I}$ in the $\mathbf{6}_{-2}$. Let us indicate this coupling by

$$
L=m_{I}(\mathcal{C}) A_{[r s]}^{I} \lambda_{\mathcal{C}}^{r} \lambda_{\mathcal{C}}^{s}
$$

where $m_{I}(\mathcal{C})$ depends on the cycle $\mathcal{C}$ wrapped by the $E D 1^{7}$.

More explicitly, given a 2-cycle $\mathcal{C}$ the 'vector' $m_{I}(\mathcal{C})$ projects on the components of $A_{[r s]}^{I}$ orthogonal to $\mathcal{C}$. This could be rephrased in more mathematical terms by interpreting $A_{[r s]}^{I}$ and $\lambda_{\mathcal{C}}^{r}$ as sections of (non-trivial) holomorphic bundles [56]. In particular, for $\mathcal{C} \approx C P^{1}$, decomposing $\left.V\right|_{\mathcal{C}}$ as

$$
\left.V\right|_{\mathcal{C}}=\sum_{i=1}^{16}\left[\mathcal{O}\left(k_{i}\right) \oplus \mathcal{O}\left(-k_{i}\right)\right]
$$

and tensoring with the spin bundle $S_{L}=\mathcal{O}(-1)$, one finds $\operatorname{dim} \operatorname{Ker}\left(\bar{\partial}_{S_{L} \otimes V}\right)=\sum_{i} k_{i}$. The integers $k_{i}$, with $k_{i} \geq 0$ without loss of generality, are further constrained by the condition $C_{2}(T)=C_{2}(V)$ on the second Chern class, that amounts to $G_{3}=d F_{3}=0$, since there are no D5-branes in the $\mathbf{T}^{6} / \mathbf{Z}_{3}$ orientifold.

Our analysis differs from [56, 30], in that we consider explicitly the coupling of the zero-modes of $\lambda_{\mathcal{C}}^{r}$ to the massless matter fields $A_{[r s]}^{I}$ in the open string spectrum. Even in the presence of a non trivial restriction to $\mathcal{C}$ of the vacuum gauge bundle $V$ one can thus have non-perturbative effects that require a field dependent pre-factor $\epsilon^{r s p q} A_{r s}^{I} A_{p q}^{J}$.

Rigid two-cycles $\mathcal{C}$ with $\sum_{i} k_{i}=4$ yield, after integrating over $\lambda$ 's and $\Theta$ 's, superpotential term of the form

$$
W_{m}=\sum_{\mathcal{C}} m_{I}(\mathcal{C}) m_{J}(\mathcal{C}) \epsilon^{r s p q} A_{r s}^{I} A_{p q}^{J}
$$

that generate a supersymmetric mass term for all the $A$ 's. Choosing a (canonical) basis of 2-cycles $\left\{\mathcal{C}_{a}\right\}$ one can expand $\mathcal{C}$ accordingly, e.g. $\mathcal{C}=\sum_{a} n^{a} \mathcal{C}_{a}$, and replace the sum over $\mathcal{C}$ with a sum over $n^{a}$. The dependence on $\mathcal{C}$ hides the dependence on the Kähler moduli $T$ 's and $Z$ 's that determine the sizes of the two-cycles. Multiple covers are related to multi-instantons and may require further investigation to be properly incorporated.

Considerations of $U(1)$ invariance suggest that each power of the (pre)factor $\epsilon^{r s p q} A_{r s}^{I} A_{p q}^{J}$ should be accompanied by a compensating factor of $F\left(Z^{\prime}, T\right) \exp (-Z / 3)$, where $F\left(Z^{\prime}, T\right)$ only depends (holomorphically) on the Kähler moduli (untwisted or twisted) which are neutral (do not shift) under the anomalous $U(1)$. This means that a mass term and a quartic term could only be generated by 'fractional' instantons. A term of the form $\operatorname{det}(A \otimes A)$ would instead require a compensating $F\left(Z^{\prime}\right) \exp (-Z)$ which can be accounted for by 'standard' ED1 instanton wrapping cycles in integral homology. Although we cannot produce a fully convincing argument, we expect these fractional branes to be allowed at the orbifold

\footnotetext{
${ }^{7} \mathrm{~A}$ formal expression for $m_{I}(\mathcal{C})$ can be obtained by a slight extension of the results in 56 .
} 
point, where the $U(1)$ appears, and to support the correct number of $\lambda$ zero-modes so as to produce the powers of $\epsilon^{r s p q} A_{r s}^{I} A_{p q}^{J}$ upon integration. Indeed, the $Z_{3}$ trapped flux in the twisted collapsed cycles generates the necessary fractional action via the $\int B \wedge C_{2} \mathrm{WZ}$ coupling of an ED3 brane wrapping a twisted four-cycle.

ED1's that wrap cycles that do not include the cycle dual to the democratic $Z$ can also contribute superpotential terms of the form

$$
W_{0}\left(Z^{\prime}\right)=\sum_{n_{a}} g\left(n_{a}\right) \exp \left(-\sum_{a} n^{a} Z_{a}^{\prime}\right)
$$

which we expect to be T-dual to the non-perturbative superpotential generated by wrapped ED3 and carefully studied in the context of toroidal orbifolds with $\Omega_{3}$ and $\Omega_{7}$ projections in [52]. As mentioned in the introduction, the conclusion of [52] is that such a superpotential combined with a flux superpotential and gaugino condensation on D7 branes can completely stabilize the closed string moduli as well as (some of) the open string moduli. Stable uplift to $\mathrm{dS}$, i.e. a positive definite square mass in the AdS ground-state, is only possible when complex structure deformations are allowed. In particular this seems to exclude the $\mathbf{T}^{6} / \mathbf{Z}_{3}$ case we focus on here. Yet inclusion of Scherk Schwarz torsion and non-geometrical fluxes, that we will discuss momentarily, and the non-perturbative superpotential discussed above allows more possibilities.

\section{Fluxes}

We will now consider the possibility of turning on closed string fluxes in the $\mathbf{Z}_{3}$ orbifold. Compatibly with the orientation projection and barring $Z_{2}$ valued fluxes and open string magnetic fluxes, the only available fluxes are the R-R 3-form flux along Re $\Omega$ or Im $\Omega$ (the real and imaginary parts of the holomorphic 3-form) and the Scherk-Schwarz torsion (metric fluxes). The flux superpotential is given by

$$
W_{\text {flux }}=\int\left(G_{3}^{R-R}-i \mathcal{T} \circ J_{C}+\mathcal{R} \bullet(* S)\right) \wedge \Omega_{3}^{C Y}
$$

where $* S$ is the 6 -dimensional dual of the dilaton 0 -form and the action of the non-geometric flux is defined as

$$
\left(\mathcal{R} \bullet A_{p}\right)_{j_{1} \cdots j_{p-3}} \equiv R^{i_{1} i_{2} i_{3}} A_{i_{1} i_{2} i_{3} j_{1} \cdots j_{p-3}}
$$

\subsection{Scherk-Schwarz Torsion on $\mathrm{Z}_{3}$ orbifold}

Let us denote the geometric torsion by $\mathcal{T}$. In a real basis it has components $\mathcal{T}_{i j}{ }^{k}$, with $i, j, k=1, \ldots 6$. In a complex basis, for compatibility with the $\mathbf{Z}_{3}$ projection, it can only have components $\mathcal{T}_{I J}{ }^{\bar{K}}=-\mathcal{T}_{J I}{ }^{\bar{K}}$ and $\overline{\mathcal{T}}_{\bar{I} \bar{J}}{ }^{K}\left(\mathcal{T}_{I J}{ }^{\bar{K}}\right)^{*}$, with $I, J, \bar{K}=1,2,3$.

The trace condition $\mathcal{T}_{i j}{ }^{i}=0$ is trivially satisfied by the allowed components.

The cocycle condition

$$
\mathcal{T}_{i j}{ }^{l} \mathcal{T}_{k l}{ }^{m}+\text { cyclic in }(i j k)=0
$$


imposes the following constraints

$$
\mathcal{T}_{I J} \bar{L}_{\overline{\mathcal{T}} \bar{L}}^{M}=0
$$

i.e. there is no further allowed cyclic permutation of the lower complex indices.

Looking at $\mathcal{T}_{I J}{ }^{\bar{L}}$ and $\overline{\mathcal{T}}_{\bar{K} \bar{L}}{ }^{M}$ as 3 complex $3 \times 3$ matrices i.e. $\mathcal{T}_{I J} \bar{L}=\left(\mathcal{T}_{I}\right) J^{\bar{L}},\left(\overline{\mathcal{T}}_{\bar{I}}\right) \bar{J}^{K}$, the constraints read

$$
\left(\mathcal{T}_{I}\right)\left(\overline{\mathcal{T}}_{\bar{J}}\right)=0
$$

Moreover antisymmetry, i.e. $\mathcal{T}_{I J}{ }^{\bar{K}}=-\mathcal{T}_{J I}{ }^{\bar{K}}$, implies the $I^{\text {th }}$ row of matrix $\mathcal{T}_{I}$ has all zero components and the $J^{\text {th }}$ row of matrix $\mathcal{T}_{I}$ has opposite components w.r.t. the $I^{\text {th }}$ row of matrix $\mathcal{T}_{J}$. Starting with the diagonal constraints (no sum over $I$ )

$$
\left(\mathcal{T}_{I}\right)\left(\overline{\mathcal{T}}_{\bar{I}}\right)=0
$$

one finds the following parametrizations for the non vanishing rows of say $\mathcal{T}_{1}$

$$
\left(\mathcal{T}_{1}\right)_{2}{ }^{\bar{K}}=\left(y_{1}, a_{1} x_{1},-x_{1}\right) \quad, \quad\left(\mathcal{T}_{1}\right)_{3}{ }^{\bar{K}}=\left(\bar{a}_{1} y_{1},\left|a_{1}\right|^{2} x_{1},-\bar{a}_{1} x_{1}\right)
$$

where $x_{1}, y_{1}, a_{1}$ are three complex numbers. Similarly

$$
\left(\mathcal{T}_{2}\right)_{1}{ }^{\bar{K}}=\left(a_{2} x_{2}, y_{2},-x_{2}\right) \quad, \quad\left(\mathcal{T}_{2}\right)_{3}{ }^{\bar{K}}=\left(\left|a_{2}\right|^{2} x_{2}, \bar{a}_{2} y_{2},-\bar{a}_{2} x_{2}\right)
$$

and finally

$$
\left(\mathcal{T}_{3}\right)_{1}{ }^{\bar{K}}=\left(a_{3} x_{3},-x_{3}, y_{3}\right) \quad, \quad\left(\mathcal{T}_{3}\right)_{2}{ }^{\bar{K}}=\left(\left|a_{3}\right|^{2} x_{3}, \bar{a}_{3} y_{3},-\bar{a}_{3} x_{3}\right)
$$

Imposing antisymmetry one can relate $x_{I}, y_{I}, a_{I}$ with $I=2,3$ to one another and to $x_{1}, y_{1}, a_{1}$, that are not constrained any further and can be used to parametrize the full solution. Dropping the index 1 for simplicity and setting $y=b x$ we find

$$
\begin{gathered}
\left(\mathcal{T}_{1}\right)_{2}{ }^{\bar{K}}=x(b, a,-1) \quad, \quad\left(\mathcal{T}_{1}\right)_{3}{ }^{\bar{K}}=x\left(\bar{a} b,|a|^{2},-\bar{a}\right) \quad, \quad\left(\mathcal{T}_{2}\right)_{1}{ }^{\bar{K}}=-x(b, a,-1) \\
\left(\mathcal{T}_{2}\right)_{3}{ }^{\bar{K}}=-x\left(|b|^{2}, a \bar{b},-\bar{b}\right) \quad, \quad\left(\mathcal{T}_{3}\right)_{1}{ }^{\bar{K}}=-x\left(\bar{a} b,|a|^{2},-\bar{a}\right) \quad, \quad\left(\mathcal{T}_{3}\right)_{2}{ }^{\bar{K}}=x\left(|b|^{2}, a \bar{b},-\bar{b}\right)
\end{gathered}
$$

that satisfy the off-diagonal $(I \neq J)$ constraints, too.

The induced superpotential (in the $D 9$-brane description) reads

$$
W_{\mathcal{T}}=\frac{V o l\left(\mathbf{T}^{6}\right)}{3} \epsilon^{I J K} \mathcal{T}_{I J}{ }^{\bar{K}} J_{K \bar{K}}
$$

and depends on all 9 untwisted complex Kähler parameters $J_{K \bar{K}}$, in fact it is simply a linear combination thereof (apart from the overall volume factor). In principle one can also consider turning-on torsion in the 'twisted' sector that would induce a dependence of the flux superpotential on the twisted Kähler moduli. Compatibility with the non vanishing magnetic flux in the open string sector remains to be investigated. 


\section{Concluding remarks}

We have derived the form of the non-perturbative and flux superpotentials for Type I strings on the $\mathbf{Z}_{3}$-orbifold after Chan-Paton symmetry breaking from $U(12) \times S O(8)$ to $U(4)_{f p} \times U(4)^{3}$ (discrete Wilson lines) or $U(4)_{f p} \times U(4)$ (continuous Wilson lines).

The determination of the precise numerical coefficients in front of the non-perturbative terms would require a very detailed analysis which is beyond the scope of the present investigation. We don't expect the qualitative structure of the non-perturbative terms to significantly change. In the case under consideration, an ADS-like superpotential is generated by ED5, reproducing 'standard' gauge instantons. Additional 'mass' terms are generated by ED1, that represent 'new' genuine stringy instantons. Closed string fluxes generate additional terms.

Before attempting a full extremization of the complete superpotential, possibly including perturbative terms that involve matter charged under the 'superconformal' $U(4)^{3}$ or $U(4)$, one has to verify compatibility of the flux superpotential and the 'instanton' superpotential. Indeed, fluxes induce non trivial warping of the geometry that may result in a 'destabilization' or 'disappearance' of the cycles wrapped by ED1's and of associate non-perturbative terms. The ADS-like superpotential, due to wrapped ED5 seems more robust, relying 'only' on the compactness of the internal manifold. It is tempting to conjecture that the combined effect of ED5 and ED1 can stabilize the open string 'moduli' and the flux superpotential can then stabilize the closed string moduli. We have only explicitly considered SS torsion in the untwisted sector but it should not be impossible to consider the effect of SS torsion in the twisted sectors.

We plan to address this and related issues in a forthcoming investigation [50]. In particular one should also analyze the Fayet-Iliopoulos D-term for the anomalous $U(1)$ that should roughly read $D=R e Z-2 A^{\dagger} A$ and try to estimate the corrections to the Kähler potential, that enters the expression of the potential [54]. In addition to supersymmetric extrema one could in fact hope to find non-supersymmetric dS (meta)stable configurations.

In a series of papers [52], superpotentials induced by fluxes and non-perturbative effects were studied in the case of orientifolds of toroidal orbifolds (including the $\mathbf{T}^{6} / \mathbf{Z}_{3}$ case at hand) with $\Omega 3 / \Omega 7$ planes and D3 and D7branes. The main conclusions were that after resolution of the orbifold geometries all (closed string) moduli can be stabilized in AdS but only very few examples, the ones with (untwisted) complex structure moduli, admit a stable uplift to DS. The inclusion of open string moduli accounting for D3/D7 brane positions, Wilson line moduli and matter fields was also analyzed in [52] but no full fledged string models with all tadpole conditions satisfied were produced.

In the present paper we have tried to partially fill in this gap and to show that the combined effect of fluxes and $E D$-brane instantons may generate interesting superpotential terms whose combined effect may well stabilize closed as well as open string moduli. In this respect it is tantalizing to observe that, at fixed closed string moduli, $W_{E D 5}(A)$ grows for small $A$ 's while $W_{E D 1}(A)$ grows at large $A$. This is admittedly very preliminary. In order to argue for complete moduli stabilization one has to perform a more detailed analysis that should also settle the issue of compatibility of the fluxes with tadpole cancellation. We leave 
this for future work [50], where we hope to address the possibility of meta-stabilization along the lines of the ISS proposal [51] that admits several realizations in string theory [53]. Another, largely unexplored contribution to the scalar potential, we plan to consider in some detail, is the D-terms from $U(1)$ R-R gauge bosons that are present in type I models with $\Omega_{5 / 9}$-planes when $h_{2,1}^{-} \neq 0$ and in models $\Omega_{3 / 7}$-planes when $h_{2,1}^{+} \neq 0$.

\section{Acknowledgments}

It is a pleasure to thank P. Anastasopoulos, R. Blumenhagen, E. Dudas, F. Fucito, S. Kovacs, D. Lust, J. F. Morales and G. Rossi for discussions. Most importantly we would like to thank G. Villadoro and F. Zwirner for collaboration in early stages and numerous enlightening discussions.

This work was supported in part by the CNRS PICS no. 2530 and 3059, INTAS grant 03-516346, MIUR-COFIN 2003-023852, NATO PST.CLG.978785, the RTN grants MRTNCT-2004-503369, EU MRTN-CT-2004-512194, MRTN-CT-2004-005104 and by a European Union Excellence Grant, MEXT-CT-2003-509661. 


\section{References}

[1] A. Sagnotti, "Open strings and their symmetry groups," [ArXiv:hep-th/0208020]. G. Pradisi and A. Sagnotti, "Open string orbifolds," Phys. Lett. B 216, 59 (1989). M. Bianchi and A. Sagnotti, "On the systematics of open string theories," Phys. Lett. B 247 (1990) 517. M. Bianchi and A. Sagnotti, "Twist symmetry and open string Wilson lines," Nucl. Phys. B 361 (1991) 519.

[2] E. Dudas, "Theory and phenomenology of type I strings and M-theory," Class. Quant. Grav. 17, R41 (2000) [ArXiv:hep-ph/0006190];

C. Angelantonj and A. Sagnotti, "Open strings," Phys. Rept. 371, 1 (2002) [Erratum-ibid. 376, 339 (2003)] [ArXiv:hep-th/0204089];

A. M. Uranga, "Chiral four-dimensional string compactifications with intersecting D-branes," Class. Quant. Grav. 20, S373 (2003) [ArXiv:hep-th/0301032];

E. Kiritsis, "D-branes in standard model building, gravity and cosmology," Fortsch. Phys. 52, 200 (2004) [Phys. Rept. 421, 105 (2005 ERRAT,429,121-122.2006)] [ArXiv:hep-th/0310001];

R. Blumenhagen, M. Cvetic, P. Langacker and G. Shiu, "Toward realistic intersecting

D-brane models," Ann. Rev. Nucl. Part. Sci. 55, 71 (2005) [ArXiv:hep-th/0502005];

R. Blumenhagen, B. Kors, D. Lust and S. Stieberger, "Four-dimensional string compactifications with D-branes, orientifolds and fluxes," [ArXiv:hep-th/0610327.

[3] C. Bachas, "A Way to break supersymmetry," [ArXiv:hep-th/9503030];

M. Berkooz, M. R. Douglas and R. G. Leigh, "Branes intersecting at angles," Nucl. Phys. B 480 (1996) 265 [ArXiv:hep-th/9606139];

C. Angelantonj, I. Antoniadis, E. Dudas and A. Sagnotti, "Type-I strings on magnetised orbifolds and brane transmutation," Phys. Lett. B 489 (2000) 223 [ArXiv:hep-th/0007090];

R. Blumenhagen, L. Goerlich, B. Kors and D. Lust, "Noncommutative compactifications of type I strings on tori with magnetic background flux," JHEP 0010 (2000) 006 [ArXiv:hep-th/0007024].

[4] I. Antoniadis, E. Kiritsis and T. N. Tomaras, "A D-brane alternative to unification," Phys. Lett. B 486 (2000) 186 [ArXiv:hep-ph/0004214];

G. Aldazabal, L. E. Ibanez, F. Quevedo and A. M. Uranga, "D-branes at singularities: A bottom-up approach to the string embedding of the standard model," JHEP 0008 (2000) 002 [ArXiv:hep-th/0005067].

[5] T. P. T. Dijkstra, L. R. Huiszoon and A. N. Schellekens, "Supersymmetric standard model spectra from RCFT orientifolds,” Nucl. Phys. B 710 (2005) 3 [ArXiv:hep-th/0411129].

[6] P. Anastasopoulos, T. P. T. Dijkstra, E. Kiritsis and A. N. Schellekens, "Orientifolds, hypercharge embeddings and the standard model," Nucl. Phys. B 759 (2006) 83 [ArXiv:hep-th/0605226].

[7] S. Kachru, M. B. Schulz and S. Trivedi, "Moduli stabilization from fluxes in a simple IIB orientifold," JHEP 0310, 007 (2003) [ArXiv:hep-th/0201028];

R. Blumenhagen, D. Lust and T. R. Taylor, "Moduli stabilization in chiral type IIB orientifold models with fluxes," [ArXiv:hep-th/0303016];

J. F. Cascales and A. M. Uranga, "Chiral $4 d N=1$ string vacua with D-branes and NSNS and RR fluxes," [ArXiv:hep-th/0303024];

P. G. Camara, L. E. Ibanez and A. M. Uranga, "Flux-induced SUSY-breaking soft terms," Nucl. Phys. B 689 (2004) 195 [ArXiv:hep-th/0311241]. F. Marchesano and G. Shiu, "MSSM 
vacua from flux compactifications," Phys. Rev. D 71, 011701 (2005) [ArXiv:hep-th/0408059] "Building MSSM flux vacua," JHEP 0411, 041 (2004) [arXiv:hep-th/0409132];

J. P. Derendinger, C. Kounnas, P. M. Petropoulos and F. Zwirner, "Superpotentials in IIA compactifications with general fluxes," Nucl. Phys. B 715 (2005) 211 [ArXiv:hep-th/0411276]. R. Blumenhagen, M. Cvetic, F. Marchesano and G. Shiu, "Chiral D-brane models with frozen open string moduli," JHEP 0503, 050 (2005) [ArXiv:hep-th/0502095];

G. Villadoro and $\mathrm{F}$. Zwirner, " $N=1$ effective potential from dual type-IIA D6/O6 orientifolds with general fluxes," ArXiv:hep-th/0503169]. J. P. Derendinger, C. Kounnas, P. M. Petropoulos and F. Zwirner, "Fluxes and gaugings: $N=1$ effective superpotentials," Fortsch. Phys. 53, 926 (2005) ArXiv:hep-th/0503229;

J. P. Conlon, F. Quevedo and K. Suruliz, "Large-volume flux compactifications: Moduli spectrum and D3/D7 soft supersymmetry breaking," JHEP 0508 (2005) 007 [ArXiv:hep-th/0505076]; J. P. Derendinger, C. Kounnas and P. M. Petropoulos, "Gaugino condensates and fluxes in $N$ = 1 effective superpotentials," Nucl. Phys. B 747 (2006) 190 [ArXiv:hep-th/0601005].

[8] M. Larosa and G. Pradisi, "Magnetized four-dimensional Z(2) $x$ Z(2) orientifolds," Nucl. Phys. B 667, 261 (2003) [ArXiv:hep-th/0305224;

I. Antoniadis and T. Maillard, "Moduli stabilization from magnetic fluxes in type I string theory," Nucl. Phys. B 716, 3 (2005) [ArXiv:hep-th/0412008];

E. Dudas and C. Timirgaziu, "Internal magnetic fields and supersymmetry in orientifolds," Nucl. Phys. B 716, 65 (2005) [ArXiv:hep-th/0502085];

M. Bianchi and E. Trevigne, "The open story of the magnetic fluxes," JHEP 0508, 034 (2005) [ArXiv:hep-th/0502147/;] "Gauge thresholds in the presence of oblique magnetic fluxes," JHEP 0601, 092 (2006) [ArXiv:hep-th/0506080];

I. Antoniadis, A. Kumar and T. Maillard, "Moduli stabilization with open and closed string fluxes, " [ArXiv:hep-th/0505260];

A. Kumar, S. Mukhopadhyay and K. Ray, "Moduli stabilization with non-Abelian fluxes," [ArXiv:hep-th/0605083].

[9] S. Kachru, R. Kallosh, A. Linde and S. P. Trivedi, "De Sitter vacua in string theory," Phys. Rev. D 68, 046005 (2003) [ArXiv:hep-th/0301240]

[10] M. Dine, N. Seiberg, X. G. Wen and E. Witten, "Non-perturbative effects on the string world-sheet" Nucl. Phys. B 278, 769 (1986);

M. Dine, N. Seiberg, X. G. Wen and E. Witten, "Non-perturbative effects on the string world-sheet. 2," Nucl. Phys. B 289, 319 (1987).

[11] K. Becker, M. Becker and A. Strominger, "Five-Branes, Membranes And Nonperturbative String Theory," Nucl. Phys. B 456, 130 (1995) [ArXiv:hep-th/9507158].

[12] R. Blumenhagen, M. Cvetic and T. Weigand, "Spacetime instanton corrections in $4 D$ string vacua - the seesaw mechanism for D-brane models," [ArXiv:hep-th/0609191];

M. Haack, D. Krefl, D. Lust, A. Van Proeyen and M. Zagermann, "Gaugino condensates and D-terms from D\%-branes," [ArXiv:hep-th/0609211,

L. E. Ibanez and A. M. Uranga, "Neutrino Majorana masses from string theory instanton effects," [ArXiv:hep-th/0609213];

B. Florea, S. Kachru, J. McGreevy and N. Saulina, "Stringy instantons and quiver gauge theories," [ArXiv:hep-th/0610003].

[13] A. Sagnotti, "A Note on the Green-Schwarz mechanism in open string theories," Phys. Lett. B 294 (1992) 196 [ArXiv:hep-th/9210127]. 
[14] L. E. Ibanez, R. Rabadan and A. M. Uranga, "Anomalous U(1)'s in type I and type IIB D= 4, $N=1$ string vacua," Nucl. Phys. B 542 (1999) 112 [ArXiv:hep-th/9808139]. M. Bianchi and J. F. Morales, "Anomalies and tadpoles," JHEP 0003 (2000) 030 [ArXiv:hep-th/0002149].

[15] L. E. Ibanez, F. Marchesano and R. Rabadan, "Getting just the standard model at intersecting branes," JHEP 0111 (2001) 002 [ArXiv:hep-th/0105155].

[16] I. Antoniadis, E. Kiritsis and J. Rizos, "Anomalous U(1)s in type I superstring vacua," Nucl. Phys. B 637 (2002) 92 [ArXiv:hep-th/0204153].

[17] P. Anastasopoulos, “4D anomalous $U(1)$ 's, their masses and their relation to $6 D$ anomalies," JHEP 0308 (2003) 005 [ArXiv:hep-th/0306042]; "Anomalous U(1)s masses in non-supersymmetric open string vacua," Phys. Lett. B 588 (2004) 119 [ArXiv:hep-th/0402105].

[18] P. Anastasopoulos, M. Bianchi, E. Dudas and E. Kiritsis, "Anomalies, anomalous U(1)'s and generalized Chern-Simons terms," JHEP 0611 (2006) 057 [ArXiv:hep-th/0605225].

[19] M. Buican, D. Malyshev, D. R. Morrison, M. Wijnholt and H. Verlinde, "D-branes at singularities, compactification, and hypercharge," [ArXiv:hep-th/0610007].

[20] P. Binetruy and E. Dudas, "Gaugino condensation and the anomalous U(1)," Phys. Lett. B 389 (1996) 503 [ArXiv:hep-th/9607172];

E. Dudas and S. K. Vempati, "Large D-terms, hierarchical soft spectra and moduli stabilisation," Nucl. Phys. B 727 (2005) 139 [ArXiv:hep-th/0506172].

[21] E. Kiritsis, "Duality and instantons in string theory," [ArXiv:hep-th/9906018].

[22] M. Billo, M. Frau, I. Pesando, F. Fucito, A. Lerda and A. Liccardo, "Classical gauge instantons from open strings," JHEP 0302, 045 (2003) [ArXiv:hep-th/0211250]; M. Billo, M. Frau, F. Fucito and A. Lerda, "Instanton calculus in $R$ - $R$ background and the topological string," JHEP 0611, 012 (2006) [ArXiv:hep-th/0606013.

[23] D. Anselmi and P. Fre, "Topological twist in four-dimensions, $R$ duality and hyperinstantons," Nucl. Phys. B 404 (1993) 288 [ArXiv:hep-th/9211121]; "Gauged hyper - instantons and monopole equations," Phys. Lett. B 347 (1995) 247 [ArXiv:hep-th/9411205]. E. Witten, "Monopoles and four manifolds," Math. Res. Lett. 1, 769 (1994) [ArXiv:hep-th/9411102].

[24] C. Angelantonj, M. Bianchi, G. Pradisi, A. Sagnotti and Y. S. Stanev, "Chiral asymmetry in four-dimensional open- string vacua," Phys. Lett. B 385 (1996) 96 [ArXiv:hep-th/9606169]; M. Cvetic, L. L. Everett, P. Langacker and J. Wang, "Blowing-up the four-dimensional Z(3) orientifold," JHEP 9904, 020 (1999) [ArXiv:hep-th/9903051].

[25] D. Amati, K. Konishi, Y. Meurice, G. C. Rossi and G. Veneziano, "Non-perturbative aspects of supersymmetric gauge theories" Phys. Rept. 162, 169 (1988);

M. A. Shifman and A. I. Vainshtein, "Instantons versus supersymmetry: Fifteen years later," hep-th/9902018.

[26] G. Veneziano and S. Yankielowicz, "An Effective Lagrangian For The Pure N=1 Supersymmetric Yang-Mills Theory," Phys. Lett. B 113, 231 (1982);

T. R. Taylor, G. Veneziano and S. Yankielowicz, "Supersymmetric QCD And Its Massless Limit: An Effective Lagrangian Analysis," Nucl. Phys. B 218, 493 (1983). 
[27] I. Affleck, M. Dine and N. Seiberg, "Supersymmetry Breaking By Instantons," Phys. Rev. Lett. 51, 1026 (1983);

I. Affleck, M. Dine and N. Seiberg, "Dynamical Supersymmetry Breaking In Supersymmetric QCD," Nucl. Phys. B 241, 493 (1984).

[28] N. Seiberg, "Electric - magnetic duality in supersymmetric nonAbelian gauge theories," Nucl. Phys. B 435, 129 (1995) [ArXiv:hep-th/9411149;

K. A. Intriligator and N. Seiberg, "Lectures on supersymmetric gauge theories and electric-magnetic duality," Nucl. Phys. Proc. Suppl. 45BC, 1 (1996) [ArXiv:hep-th/9509066.

[29] L. J. Dixon, V. Kaplunovsky and J. Louis, "Moduli dependence of string loop corrections to gauge coupling constants," Nucl. Phys. B 355, 649 (1991);

I. Antoniadis, E. Gava and K. S. Narain, "Moduli Corrections To Gauge And Gravitational Couplings In Four-Dimensional Superstrings,” Nucl. Phys. B 383 (1992) 93

[ArXiv:hep-th/9204030];

E. Kiritsis and C. Kounnas, "Infrared Regularization Of Superstring Theory And The One Loop Calculation Of Coupling Constants," Nucl. Phys. B 442 (1995) 472

[ArXiv:hep-th/9501020]; Nucl. Phys. Proc. Suppl. 41 (1995) 331 [ArXiv:hep-th/9410212].

[30] E. Silverstein and E. Witten, "Criteria for conformal invariance of (0,2) models," Nucl.

Phys. B 444, 161 (1995) [ArXiv:hep-th/9503212;

C. Beasley and E. Witten, "New instanton effects in string theory," JHEP 0602 (2006) 060 [ArXiv:hep-th/0512039].

[31] C. Bachas, C. Fabre, E. Kiritsis, N. A. Obers and P. Vanhove, "Heterotic/type-I duality and D-brane instantons," Nucl. Phys. B 509 (1998) 33 [ArXiv:hep-th/9707126];

E. Kiritsis and N. A. Obers, "Heterotic/type-I duality in D i 10 dimensions, threshold corrections and D-instantons," JHEP 9710 (1997) 004 [ArXiv:hep-th/9709058];

C. Bachas, "Heterotic versus type I," Nucl. Phys. Proc. Suppl. 68 (1998) 348 [ArXiv:hep-th/9710102].

[32] N. Seiberg and E. Witten, "Electric - magnetic duality, monopole condensation, and confinement in N=2 supersymmetric Yang-Mills theory," Nucl. Phys. B 426, 19 (1994) [Erratum-ibid. B 430, 485 (1994)] [ArXiv:hep-th/9407087];

N. Seiberg and E. Witten, "Monopoles, duality and chiral symmetry breaking in N=2 supersymmetric QCD," Nucl. Phys. B 431, 484 (1994) [ArXiv:hep-th/9408099].

[33] M. Bianchi, M. B. Green, S. Kovacs and G. Rossi, "Instantons in supersymmetric Yang-Mills and D-instantons in IIB superstring theory," JHEP 9808, 013 (1998)

[ArXiv:hep-th/9807033];

N. Dorey, T. J. Hollowood, V. V. Khoze and M. P. Mattis, "The calculus of many instantons," Phys. Rept. 371, 231 (2002) [ArXiv:hep-th/0206063];

S. Kovacs, "On instanton contributions to anomalous dimensions in $N=4$ supersymmetric Yang-Mills theory," Nucl. Phys. B 684, 3 (2004) [ArXiv:hep-th/0310193].

[34] M. F. Atiyah, N. J. Hitchin, V. G. Drinfeld and Yu. I. Manin, "Construction of instantons," Phys. Lett. A 65, 185 (1978).

[35] M. R. Douglas, "Branes within branes," [ArXiv:hep-th/9512077].

[36] D. S. Freed and E. Witten, "Anomalies in string theory with D-branes," [ArXiv:hep-th/9907189]. 
[37] A. K. Kashani-Poor and A. Tomasiello, "A stringy test of flux-induced isometry gauging," Nucl. Phys. B 728, 135 (2005) [ArXiv:hep-th/0505208].

[38] M. Marino, R. Minasian, G. W. Moore and A. Strominger, "Nonlinear instantons from supersymmetric p-branes," JHEP 0001, 005 (2000) [ArXiv:hep-th/9911206].

[39] T. W. Grimm and J. Louis, "The effective action of $N=1$ Calabi-Yau orientifolds," Nucl. Phys. B 699 (2004) 387 [ArXiv:hep-th/0403067].

[40] P. Candelas, E. Derrick and L. Parkes, "Generalized Calabi-Yau manifolds and the mirror of a rigid manifold," Nucl. Phys. B 407 (1993) 115 [ArXiv:hep-th/9304045].

[41] Y. E. Antebi, Y. Nir and T. Volansky, "Solving flavor puzzles with quiver gauge theories," Phys. Rev. D 73, 075009 (2006) [ArXiv:hep-ph/0512211].

[42] D. Berenstein, "Branes vs. GUTS: Challenges for string inspired phenomenology," [ArXiv:hep-th/0603103].

[43] D. Berenstein and S. Pinansky, "The minimal quiver standard model," [ArXiv:hep-th/0610104].

[44] M. Bianchi, G. Pradisi and A. Sagnotti, "Toroidal compactification and symmetry breaking in open string theories," Nucl. Phys. B 376 (1992) 365;

M. Bianchi, "A note on toroidal compactifications of the type I superstring and other superstring vacuum configurations with 16 supercharges," Nucl. Phys. B 528, 73 (1998) [ArXiv:hep-th/9711201].

[45] M. Cvetic and P. Langacker, " $D=4 N=1$ type IIB orientifolds with continuous Wilson lines, moving branes, and their field theory realization," Nucl. Phys. B 586, 287 (2000) [ArXiv:hep-th/0006049];

M. Cvetic, A. M. Uranga and J. Wang, "Discrete Wilson lines in $N=1 \mathrm{D}=4$ type IIB orientifolds: A systematic exploration for Z(6) orientifold," Nucl. Phys. B 595, 63 (2001) [ArXiv:hep-th/0010091].

[46] I. Antoniadis, E. Dudas and A. Sagnotti, "Supersymmetry breaking, open strings and M-theory," Nucl. Phys. B 544 (1999) 469 [ArXiv:hep-th/9807011].

[47] I. Antoniadis, C. Bachas and E. Dudas, "Gauge couplings in four-dimensional type I string orbifolds," Nucl. Phys. B 560 (1999) 93 [ArXiv:hep-th/9906039];

D. Lust and S. Stieberger, "Gauge threshold corrections in intersecting brane world models," [ArXiv:hep-th/0302221].

[48] P. Anastasopoulos, M. Bianchi, G. Sarkissian and Y. S. Stanev, "On gauge couplings and thresholds in Type I Gepner models and otherwise," [ArXiv:hep-th/0612234].

[49] N. Akerblom, R. Blumenhagen, D. Lust, E. Plauschinn and M. Schmidt-Sommerfeld, "Non-perturbative SQCD superpotentials from string instantons," [ArXiv:hep-th/0612132].

[50] M. Bianchi, E. Kiritsis, G. Villadoro and F. Zwirner, work in progress.

[51] K. Intriligator, N. Seiberg and D. Shih, "Dynamical SUSY breaking in meta-stable vacua," JHEP 0604, 021 (2006) [ArXiv:hep-th/0602239].

[52] D. Lust, S. Reffert, E. Scheidegger, W. Schulgin and S. Stieberger, "Moduli stabilization in type IIB orientifolds. II," [ArXiv:hep-th/0609013];

D. Lust, S. Reffert, E. Scheidegger and S. Stieberger, "Resolved toroidal orbifolds and their orientifolds," ArXiv:hep-th/0609014]. 
[53] S. Franco, I. Garcia-Etxebarria and A. M. Uranga, "Non-supersymmetric meta-stable vacua from brane configurations," [ArXiv:hep-th/0607218. R. Argurio, M. Bertolini, S. Franco and S. Kachru, "Gauge/ gravity duality and meta-stable dynamical supersymmetry breaking," [ArXiv:hep-th/0610212].

[54] G. Villadoro and F. Zwirner, "de Sitter vacua via consistent D-terms," Phys. Rev. Lett. 95 (2005) 231602 [ArXiv:hep-th/0508167]; "D terms from D-branes, gauge invariance and moduli stabilization in flux compactifications," JHEP 0603 (2006) 087 [ArXiv:hep-th/0602120].

[55] M. Dine, N. Seiberg and E. Witten, "Fayet-Iliopoulosterms in string theory" Nucl. Phys. B 289, 589 (1987).

[56] E. Witten, "World-sheet corrections via D-instantons," JHEP 0002 (2000) 030 [ArXiv:hep-th/9907041]. 